\title{
Electron acceleration in solar flares: theory of spectral evolution
}

\author{
P. C. Grigis and A. O. Benz
}

\author{
Institute of Astronomy, ETH Zurich, 8092 Zurich, Switzerland \\ e-mail:pgrigis@astro.phys.ethz.ch
}

Received 12 June 2006 / Accepted 31 July 2006

\begin{abstract}
Context. Stochastic acceleration is thought to be a key mechanism in the energization of solar flare electrons.

Aims. We study whether stochastic acceleration can reproduce the observed soft-hard-soft evolution of the spectral features of the hard X-ray emitted by suprathermal electrons. We pay special attention to the effects of particle trapping and escape.

Methods. The Fokker-Planck equation for the electron distribution is integrated numerically using the coefficients derived by Miller et al. for transit-time damping acceleration. The electron spectra are then converted to photon spectra for comparison with RHESSI observations of looptop sources.

Results. The presence of particle escape softens the model spectra computed in the stochastic acceleration framework. The ratio between the efficiency of trapping and acceleration controls the spectral evolution which follows a soft-hard-soft pattern. Furthermore, a pivot point (that is, a common crossing point of the accelerated particle spectra at different times) is found at around $10 \mathrm{keV}$. It can be brought into agreement with the observed value of $20 \mathrm{keV}$ by enhanced trapping through an electric potential.

Conclusions. The model proposed here accounts for the key features observed in the spectral evolution of hard X-ray emission from looptop sources.
\end{abstract}

Key words. Sun: flares - Sun: X-rays, gamma rays - acceleration of particles

\section{Introduction}

Solar flares emit large amounts of continuum hard X-ray bremsstrahlung from energetic electrons up to several hundreds of $\mathrm{keV}$. The thermal energy of the ambient electrons in the corona is substantially lower, with electron temperatures observed up to $6 \mathrm{MK}$ in nonflaring active regions (Brosius et al. 1996; Benz \& Grigis 2002). Clearly, a mechanism is needed to accelerate the electrons out of the thermal distribution up to relativistic energies. More than $10^{31}$ erg (Lin et al. 2003; Emslie et al. 2004; Kane et al. 2005) of energy can be transferred into non-thermal electrons during a major solar flare over a period of a few hundred seconds.

These numbers set stringent requirements to acceleration models, and it is by no means a simple task to identify the physical processes involved. Most of the proposed models fall into three broad classes: electric DC field acceleration, stochastic acceleration and shock acceleration (see e.g. the review by Aschwanden 2002).

Miller et al. (1996, hereafter MLM) proposed a stochastic acceleration mechanism where electrons are energized by small amplitude turbulent fast-mode waves, the transit-time damping model. MLM showed that their model could successfully account for the observed number and energy of electrons accelerated above $20 \mathrm{keV}$ in subsecond spikes or energy release fragments in impulsive solar flares. However, they made no attempt to explain the observed hard X-ray spectra (which are softer than predicted by the transit-time damping model) and did not consider spectral evolution. The MLM approach does not account for escape and transport processes.

What is the effect of escape on the electron spectrum in the accelerator? In stochastic acceleration, each electron describes a random walk in energy space. The effect of escape is to shorten the dwell time of the particles in the accelerator, and therefore it limits the average energy of the electrons. This results in a softer spectrum. Therefore, we need to take in account escape to compare quantitatively the model predictions with hard X-ray observations. Furthermore, transport effects account for the modification of the electron spectrum from the time they leave the accelerator until they reach the hard X-ray emitting regions. The usual interpretation is that the particles are accelerated near the top of magnetic loops, but most of the X-rays are emitted in the loop footpoints, where the density is much larger. Nevertheless, looptop sources are also observed (Masuda et al. 1994).

Observations from the Reuven Ramaty High Energy Imaging Spectrometer (RHESSI, Lin et al. 2002) deliver hard X-ray spectra of both footpoint and looptop sources (Emslie et al. 2003; Liu et al. 2004). Looptop sources are better suited for comparison with accelerator models than footpoint sources, since one does not need to take into account the spatial transport from the accelerator. The emission from the modeled electron distribution can be directly compared with the observed spectra of the looptop source.

Battaglia \& Benz (2006) present a careful and comprehensive study of the spectral evolution of looptop and footpoint sources for 5 well observed RHESSI flares. They find that looptop sources systematically show the soft-hard-soft (SHS) behavior observed spectroscopically for the total flare emission (Grigis \& Benz 2004, and references therein). On the other hand, some footpoint sources do not follow the SHS pattern. These observations suggest that the SHS behaviour is a property of the acceleration process.

The observed SHS behavior manifests itself as a tight correlation between the curves describing the time evolution of the negative spectral index $\gamma$ and the normalization $J_{E_{0}}$ of the 
Table 1. Observational constraints for looptop sources (taken from Battaglia \& Benz 2006).

\begin{tabular}{lll}
\hline \hline Parameter description & Average & Range \\
\hline Pivot-point energy $\varepsilon_{*}$ & $20 \mathrm{keV}$ & $16-24 \mathrm{keV}$ \\
Pivot-point flux ${ }^{a} J_{*}$ & 2 & $1-4$ \\
Mean temperature & 22 & $18-25 \mathrm{MK}$ \\
Nonthermal fitting range: & & \\
$\quad$ Lower energy $\varepsilon_{\min }$ & $25 \mathrm{keV}$ & $20-30 \mathrm{keV}$ \\
$\quad$ Upper energy $\varepsilon_{\max }$ & $60 \mathrm{keV}$ & $40-80 \mathrm{keV}$ \\
\hline
\end{tabular}

${ }^{a}$ In units of photons $\mathrm{cm}^{-2} \mathrm{~s}^{-1} \mathrm{keV}^{-1}$.

non-thermal power-law component of the photon spectra, measured at a fixed reference energy $E_{0}$. RHESSI observations show a linear relation between $\gamma$ and $\log J_{E_{0}}$ (Grigis \& Benz 2004). This is equivalent (Grigis \& Benz 2005) to the presence of a pivot point, that is, a common point where the power-law component of the photon spectra observed at different times intersect. Therefore, the position of the pivot point can be found observationally by fitting the regression line to $\gamma$ and $\log J_{E_{0}}$. Since the observations show that during the impulsive phase of most flares the scatter around such a model is relatively small, such fittings provide us with two new meaningful observational parameters which can be used for comparison with theories. The best-fit pivot-point energy can be measured by RHESSI with an accuracy of 10-20\% (Battaglia \& Benz 2006).

The measured energies of the pivot points lie in the range of 18-24 keV for looptop sources and 13-15 keV for footpoint sources. In Table 1 we summarize the observational results from Battaglia \& Benz (2006) which we will use to constrain the transit-time damping acceleration model.

In this paper we study how well the transit-time damping model endowed with an escape mechanism can account for the hard X-ray spectra of the looptop sources seen by RHESSI and, in particular, for their spectral evolution. We investigate whether the spectrum of the photons emitted by the modeled electrons in the accelerator shows SHS behavior and how well this quantitatively agrees with the observations.

We proceed as follows: a modified version of the MLM model with a better characterization of electron trapping is presented in Sect. 3. The evolution of the electron energy distribution function is given by a diffusion equation which is integrated numerically and transformed into photon spectra. In Sect. 4 we present the resulting photon spectra and compare their behaviour with the observations. The results are discussed in Sect. 5 and conclusions are drawn in Sect. 6.

\section{Pivot-point theory}

In this section the mathemathical foundations needed for the comparison of the model results with the observations are presented. In particular we explain what are the consequences of the correlation in time between flux and spectral index. By the introduction of a new parameter, the pivot-point, the time dependence can be eliminated, thus simplifying the analysis.

The theory is presented in a general context, and can be applied to any kind of spectra (for example, photons or electrons) described by a power-law in some energy range. The starting point of this analysis is the presence of a correlation between the $\operatorname{logarithm}$ of the flux, $\log F_{E_{0}}$, measured at energy $E_{0}$ and the spectral index $\delta$, where the time dependent spectrum is given by

$F(E, t)=F_{E_{0}}(t)\left(\frac{E}{E_{0}}\right)^{-\delta(t)}$,

for $t$ in a time interval $T_{\mathrm{INT}}$ (for instance, the duration of a flare emission peak). Panel (a) of Fig. 1 shows the ideal case where the correlation in time between $\delta$ (lower curve) and $\log F_{E_{0}}$ (upper curve) is perfect: the two curves are parallel. In such a case, a plot of $\delta$ vs. $\log F_{E_{0}}$ is linear. This is shown in panel (c). The relation between these two parameters can be expressed as

$\delta(t)=a \cdot \log F_{E_{0}}(t)+b$,

where $a \neq 0$, and the parameters $a$ and $b$ do not depend on time.

Some points with selected values of $\delta$ are marked by special symbols in Fig. 1: circles for $\delta=2$, triangles for $\delta=4$, squares for $\delta=6$ and stars for $\delta=8$. In panel (b) the spectra corresponding to these values of the spectral index are plotted together. It is evident that all these spectra intersect in a common point, the pivot point. The coordinates of the pivot point are defined as $\left(E_{*}, F_{*}\right)$. The presence of a linear relation between $\delta(t)$ and $\log F_{E_{0}}(t)$ is equivalent to the presence of a pivot-point. This means that panel (a) in Fig. 1 implies both (b) and (c). A detailed proof follows.

\subsection{Proof of the equivalence between pivot point and correlation of $\delta$ with $\log F$}

The following statements are equivalent:

(i) There is an $E_{*} \neq E_{0}$ such that $F\left(E_{*}, t_{1}\right)=F\left(E_{*}, t_{2}\right)=: F_{*}$ for all $t_{1}, t_{2} \in T_{\mathrm{INT}}$.

(ii) There exist constants $a \neq 0$ and $b$ such that

$$
\begin{aligned}
& \delta(t)=a \log F_{E_{0}}(t)+b \\
& \text { for all } t \in T_{\mathrm{INT}} .
\end{aligned}
$$

The parameters $a$ and $b$ are given by

$$
\begin{aligned}
& a=\frac{1}{\log \left(E_{*} / E_{0}\right)}, \\
& b=\frac{-\log F_{*}}{\log \left(E_{*} / E_{0}\right)} .
\end{aligned}
$$

We give first the proof that (i) $\Longrightarrow$ (ii). Because $F\left(E_{*}, t\right)=F_{*}$ for all $t \in T_{\text {INT }}$, Eq. (1) yields

$\log F_{*}=\log \left[F_{E_{0}}\left(\frac{E_{*}}{E_{0}}\right)^{-\delta}\right]=\log F_{E_{0}}-\delta \log \left(E_{*} / E_{0}\right)$

which can be solved for $\delta$, yielding

$$
\begin{aligned}
\delta & =\frac{\log F_{E_{0}}}{\log \left(E_{*} / E_{0}\right)}-\frac{\log F_{*}}{\log \left(E_{*} / E_{0}\right)} \\
& =a \log F_{E_{0}}+b,
\end{aligned}
$$

where $a \neq 0$ for finite values of $E_{*}, E_{0}$.

Now we prove (ii) $\Longrightarrow$ (i). Let us define $E_{*}=E_{0} \exp (1 / a) \neq E_{0}$. Then using Eqs. (1), (4) and (3) we get

$$
\begin{aligned}
\log F\left(E_{*}\right) & =\log F_{E_{0}}-\delta \cdot \log \left(\frac{E_{*}}{E_{0}}\right) \\
& =\log F_{E_{0}}-\frac{\delta}{a} \\
& =-\frac{b}{a}
\end{aligned}
$$



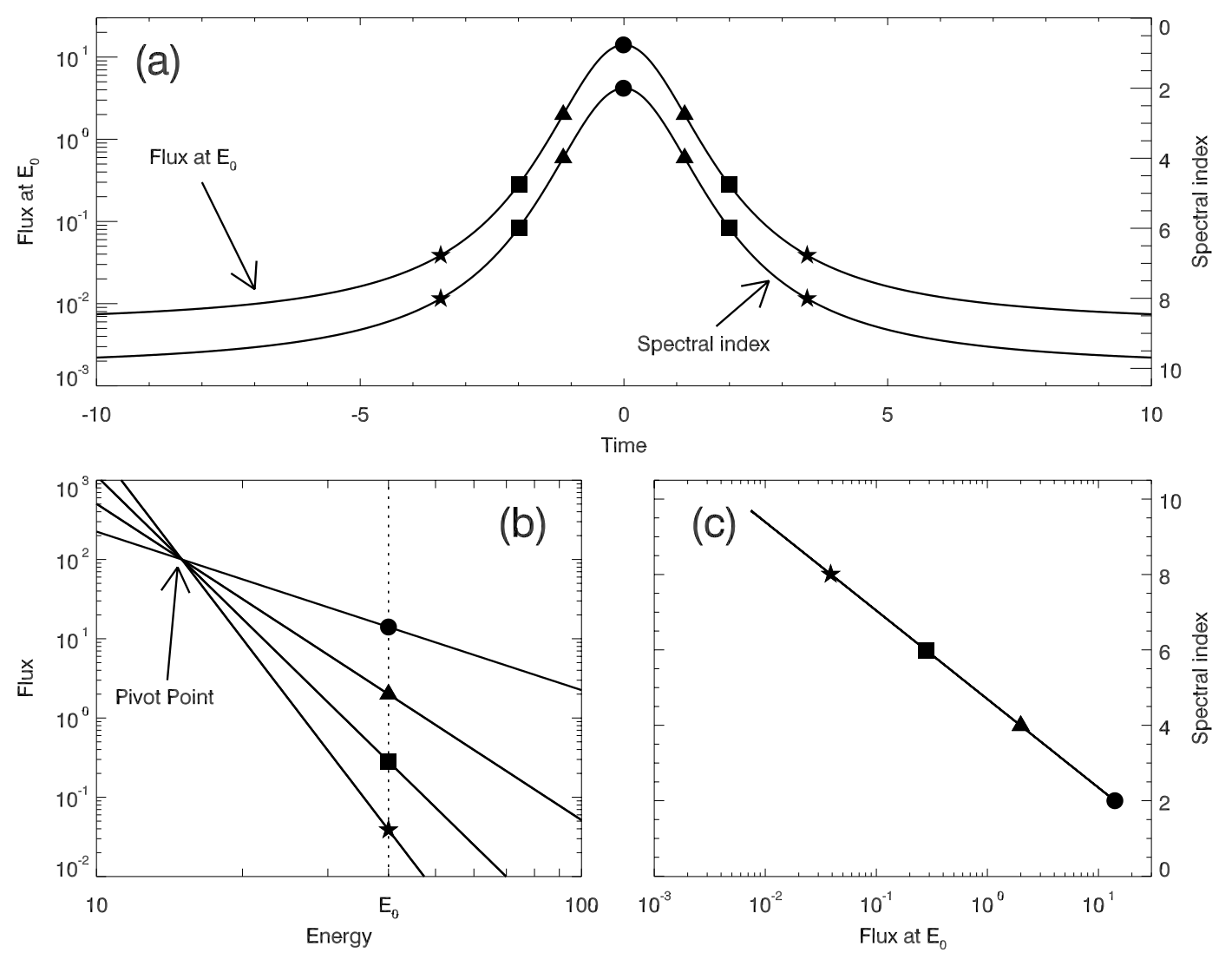

Fig. 1. Schematic illustration of the basic properties of a spectral evolution. Panel a): time evolution of the logarithm of the flux measured at reference energy $E_{0}$ (upper curve) and of the spectral index (lower curve) in the case where they are perfectly correlated. Panel b): spectra for different values of the spectral index, crossing at the pivot point. Panel c): linar dependence of the spectral index on the logarithm of the flux. In all panels, the stars, squares, triangles and circles mark points with spectral indices of, respectively, 8, 6, 4 and 2.

which does not depend on $\delta$ or $F_{E_{0}}$. Therefore, $\left(E_{*}, F_{*}\right)$ are the coordinates of the pivot point. Q.E.D.

\subsection{Implications}

We have established above formally the equivalence between the presence of a pivot point and the linear relation between the spectral index and the logarithm of the flux measured at some fixed energy. We note that the shape of the curves describing the time evolution of $\delta$ and $F_{E_{0}}$ does not influence the results described above. The time dependence can be eliminated: this simplifies the analysis and allows the new parameter, the pivotpoint position, to be used in the comparison of observations with theoretical models. Conversely, if the observation show that $\delta$ and $\log F_{E_{0}}$ do not correlate well in time, we will not find any pivot-point like behavior in the spectral evolution.

Our derivation assumes that the spectra are represented by power-laws at all energies. In this case, at energies lower than the pivot-point energy, the linear relation between flux and spectral index changes sign and higher fluxes would correspond to steeper spectra instead of harder ones. However, the observed spectra are not given by power-laws at low energies, because steep spectra are not integrable and therefore would imply an infinite number of particles, which is not physically possible. Therefore a deviation from the power-law form is expected in the form of a turnover at low energies (Saint-Hilaire \& Benz 2005; Sui et al. 2005). This means that it is well possible that the spectra do not extend all the way to the pivot point energy, even if a correlation between the spectral index and the flux is observed at higher energies. In such a situation the pivot point is virtual, in the sense that its flux is higher than the spectral flux at the same energy. In this case the analysis given above retain its validity, but the spectra used should be understood as the power-law extensions of the physical spectra. Such a situation is represented in Fig. 2.

We emphasize that the presence of a pivot-point is a local property of a family of curves at energy $E_{0}$, in the sense that it can be seen as a convergence point for the tangents in $E_{0}$ to the family of curves (in logarithmic space). For power-law functions, the coordinates of the pivot point are independent of the energy $E_{0}$, because their logarithmic derivative is constant as a function of energy. Other functions exist for which there is a different pivot point for each value of $E_{0}$.

For the sake of completeness, we report here the differential equation whose general solution is the family of curves yielding a pivot point (in the sense of a convergence point for the tangents in linear space) in $x^{*}(x), y^{*}(x)$ :

$\frac{\mathrm{d} y}{\mathrm{~d} x}+\frac{y}{x^{*}(x)-x}=\frac{y^{*}(x)}{x^{*}(x)-x}$

where in our case, $x=\log E, x^{*}=\log E^{*}, y=\log F$ and $y^{*}=$ $\log F^{*}$.

In the special case $x^{*}=$ const. and $y^{*}=$ const. (that is, a unique pivot point for all energies), the general solution of Eq. (12) is given by $y-y^{*}=m \cdot\left(x-x^{*}\right)$, the family of all straight lines intersecting at $x^{*}$ and $y^{*}$. 


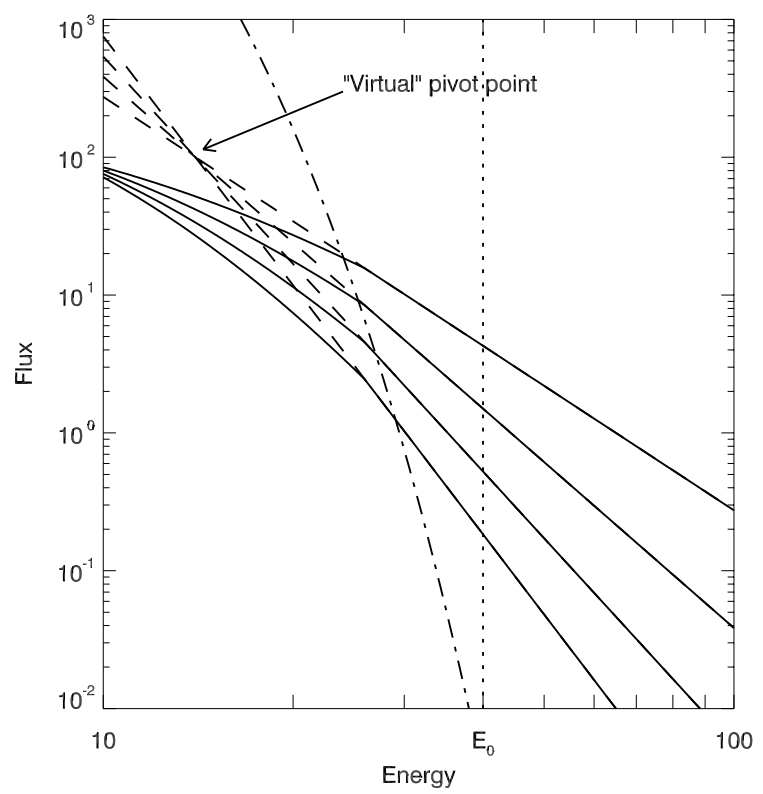

Fig. 2. Spectra (mock data, continuous lines) turning over at low energy. The power-law extensions of the spectra (dashed lines) intersect in a virtual pivot point, with larger flux than the one from the spectra. The dashed-dotted line represent an additional thermal component, effectively hiding the spectral behavior at low energies.

\section{The model}

The transit-time damping model is described in great detail by MLM. Here we first summarize the important features of the model presented by MLM, and then describe our modification of the model.

\subsection{The transit-time damping model}

The source of the flare energy is an event of magnetic reconnection, where magnetic field energy is transferred, by an unspecified mechanism, into small-amplitude magnetoacoustic fast mode waves. These waves have very large wavelengths, comparable with the length scale of the global magnetic field restructuring induced by the reconnection process.

A cascading process then takes place, producing waves with larger wave vector $k$ and isotropic distribution. This process transfers wave energy density from the low $k$ into the large $k$ waves. The electrons interact with the parallel component of the magnetic field of the large $k$ waves. An electron of velocity $v$ can efficiently exchange energy with the waves if the resonance condition $k_{\|} v_{\|}=\omega=k v_{\mathrm{A}}$ is satisfied, where the subscript $\|$ denotes the component parallel to the ambient magnetic field, $\omega$ is the wave frequency and $v_{\mathrm{A}}$ is the Alfvén speed. The same condition can also be expressed as $\mu \eta v=v_{\mathrm{A}}$, where $\mu$ and $\eta$ are the cosines of the angle between the ambient magnetic field and the direction of propagation of the electrons and the waves, respectively. From this form of the equation it is clear that only electrons faster then $v_{\mathrm{A}}$ can be accelerated by this mechanism. The perpendicular velocity $v_{\perp}$ is not affected by these interactions.

Since the acceleration only occurs in the direction of the ambient magnetic field, the electron distribution becomes strongly elongated in parallel direction in the absence of pitch-angle scattering. This reduces considerably the efficiency of transit-time damping acceleration, because for fast particle with $v \gg v_{\mathrm{A}}$ and $|\mu| \approx 1$ the resonance condition can only be satisfied by waves with $|\eta|<v_{\mathrm{A}} / v$, which represent only a small fraction of all the
Table 2. Description of the parameters used in the model.

\begin{tabular}{lll}
\hline \hline Param. & Description & Default value \\
\hline$B$ & Ambient magnetic field strength & $500 \mathrm{G}$ \\
$n$ & electron density & $10^{10} \mathrm{~cm}^{-3}$ \\
$T$ & Ambient plasma temperature & $10 \mathrm{MK}$ \\
$\log \Lambda$ & Coulomb logarithm & 18 \\
$\Omega_{\mathrm{H}}$ & Proton gyrofrequency & $4.78 \mathrm{MHz}$ \\
$T_{\mathrm{H}}$ & Time unit $\Omega_{\mathrm{H}}^{-1}$ & $2.09 \times 10^{-7} \mathrm{~s}$ \\
$v_{\mathrm{A}}$ & Alfvén speed & $0.034 \mathrm{c}$ \\
$U_{\mathrm{T}}$ & Energy density of the accelerating & \\
& waves & $2 \mathrm{erg} \mathrm{cm} \mathrm{cm}^{-3}$ \\
$U_{\mathrm{B}}$ & Energy density of the ambient & \\
& field $\left(\frac{1}{8} B^{2} / \pi\right)$ & $10^{4} \mathrm{erg} \mathrm{cm}^{-3}$ \\
$\langle k\rangle$ & Average wavenumber of the & $4.8 \times 10^{-4} \mathrm{~cm}^{-1}$ \\
& turbulence & $($ free $)$ \\
$I_{\mathrm{ACC}}$ & Acceleration parameter: & \\
& $I_{\mathrm{ACC}}=\frac{U_{\mathrm{T}}}{U_{\mathrm{B}}} \cdot \frac{c\langle k\rangle}{\Omega_{\mathrm{H}}}$ & $($ free) \\
$\tau$ & Escape time & \\
\hline
\end{tabular}

available phase space. The acceleration efficiency can therefore be increased by the presence of a mechanism which isotropizes the electron distribution, allowing particles with small $|\mu|$ to interact with waves with $|\eta|>v_{\mathrm{A}} / v$. MLM assume that an isotropization mechanism is present, but do not address the details of the process.

The model described by MLM is self consistent in the sense that it describes the evolution in time of both the electrons (which are accelerated) and the waves (which are damped). The efficiency of the electron acceleration by the waves is determined by the total energy density of the waves $U_{\mathrm{T}}$ and by the shape of the wave spectrum: large $k$ waves exchange energy with the electrons faster because the transit time between the magnetic perturbations gets shorter. The coefficients describing the diffusion of the electrons in energy space depend on the product of $U_{\mathrm{T}}$ and $\langle k\rangle$, where the latter represents the average wave-vector of the waves.

The evolution of the electrons is described by the FokkerPlanck equation for the electron distribution in energy space. MLM consider the effects of the acceleration by the waves and of collisions with the ambient plasma.

\subsection{Transit-time damping and escape}

In this paper, we study the electron and photon spectra produced by a simplified transit-time damping acceleration model. We do not address here the problem of the time it takes for the accelerated electron distribution to reach equilibrium, considering that MLM have established this time to be sufficiently short. Therefore we need not to account explicitely for the MHD cascading process, but we will instead keep $U_{\mathrm{T}} \cdot\langle k\rangle$ as a free parameter of our model. This means that only one partial differential equation describes the time-evolution of the system instead of a set of two coupled equations.

A short description of the physical parameters upon which the model depends is given in Table 2, where a default reference value for each parameter is listed. In the following these default values will be implied if different values are not explicitely mentioned.

The transit-time damping acceleration model describes the evolution of the electron population in energy and time. We denote by $N(E) \mathrm{d} E$ the number of electrons per cubic centimeter with energy in the interval $\mathrm{d} E$ around $E$, where $E$ is the 
dimensionless particle energy in units of $m_{\mathrm{e}} c^{2}$. The total electron density is $n=\int_{0}^{\infty} N(E) \mathrm{d} E$. The evolution in time of the distribution function $N(E, t)$ is given by the convective-diffusive equation

$$
\begin{aligned}
\frac{\partial N}{\partial t}= & \frac{1}{2} \frac{\partial^{2}}{\partial E^{2}}\left[\left(D_{\mathrm{COLL}}+D_{\mathrm{T}}\right) N\right]-\frac{\partial}{\partial E}\left[\left(A_{\mathrm{COLL}}+A_{\mathrm{T}}\right) N\right] \\
& -S(E) \cdot N+Q(E) .
\end{aligned}
$$

The coefficients $D_{\mathrm{T}}$ and $A_{\mathrm{T}}$ describe the diffusion and convection in energy space due to the interaction of the electrons with the waves. The coefficients $D_{\mathrm{COLL}}$ and $A_{\mathrm{COLL}}$ represent the effect that collisions against the ambient plasma have on the distribution function. We include the effects of the escape by a sink term $S(E)$ and a source term $Q(E)$ described below.

The acceleration coefficients are given by MLM, and we report them here written in dimensionless form:

$$
\begin{aligned}
& A_{\mathrm{T}}(E)=\frac{\pi}{4} \beta_{\mathrm{A}}^{2} I_{\mathrm{ACC}} \gamma \beta g(\beta) \\
& D_{\mathrm{T}}(E)=\frac{\pi}{8} \beta_{\mathrm{A}}^{2} I_{\mathrm{ACC}} \gamma^{2} \beta^{3} f(\beta)
\end{aligned}
$$

where

$$
\begin{aligned}
\gamma & =1+E, \quad \beta=\sqrt{1-\gamma^{-2}}, \quad \xi=\frac{\beta_{\mathrm{A}}}{\beta} \\
f(\beta) & =-1.25-\left(1+2 \xi^{2}\right) \log \xi+\xi^{2}+0.25 \xi^{4} \\
g(\beta) & =\frac{1}{4 \gamma^{2}}\left(4 \xi^{2} \log \xi-\xi^{4}+1\right)+f(\beta) .
\end{aligned}
$$

The acceleration coefficients vanish for $E<E_{\mathrm{A}}$, where $E_{\mathrm{A}}$ is the kinetic energy of a particle with speed $\beta_{\mathrm{A}}=v_{\mathrm{A}} / c$, because the resonance condition cannot be satisfied below the Alfvén speed.

The coefficients for Coulomb collisions of the accelerated particles with the thermal background population (Spitzer 1962; Trubnikov 1965) are given by:

$$
\begin{aligned}
& A_{\mathrm{COLL}}=-v n\left[\psi(x)-\psi^{\prime}(x)\right] E^{-1 / 2} \\
& D_{\mathrm{COLL}}=2 v n \frac{\psi(x)}{x} E^{1 / 2}
\end{aligned}
$$

with

$$
\begin{aligned}
& x=\frac{m_{\mathrm{e}} c^{2} E}{k_{\mathrm{B}} T}, \quad v=\frac{\sqrt{8} \pi e^{4} \log \Lambda T_{\mathrm{H}}}{m_{\mathrm{e}}^{2} c^{3}} \\
& \psi(x)=2 \pi^{-1 / 2} \int_{0}^{x} \sqrt{t} \mathrm{e}^{-t} \mathrm{~d} t .
\end{aligned}
$$

The energy dependence of the acceleration and collisional coefficients is shown in Fig. 3.

The sink term is given by:

$S(E)=\frac{T_{\mathrm{H}} \beta}{\tau}$,

where $\tau$ is the escape time. This represents a purely kinematic escape for a spatially homogeneous electron distribution (leakybox escape). We can understand this term in the following way: let's assume that the plasma is contained in a cylinder with length $L$ and cross section $A$, having a spatially homogeneous electron density $n$ with a total number of particles $N_{\text {TOT }}=\int n \mathrm{~d} V=$ $n \cdot L \cdot A$ all having the same speed $v$. After a time interval $\mathrm{d} t$, the number of particles which have escaped the cylinder (assuming free streaming without collisions) is $\mathrm{d} N_{\mathrm{TOT}}=n \cdot v \cdot A \cdot \mathrm{d} t$. The rate
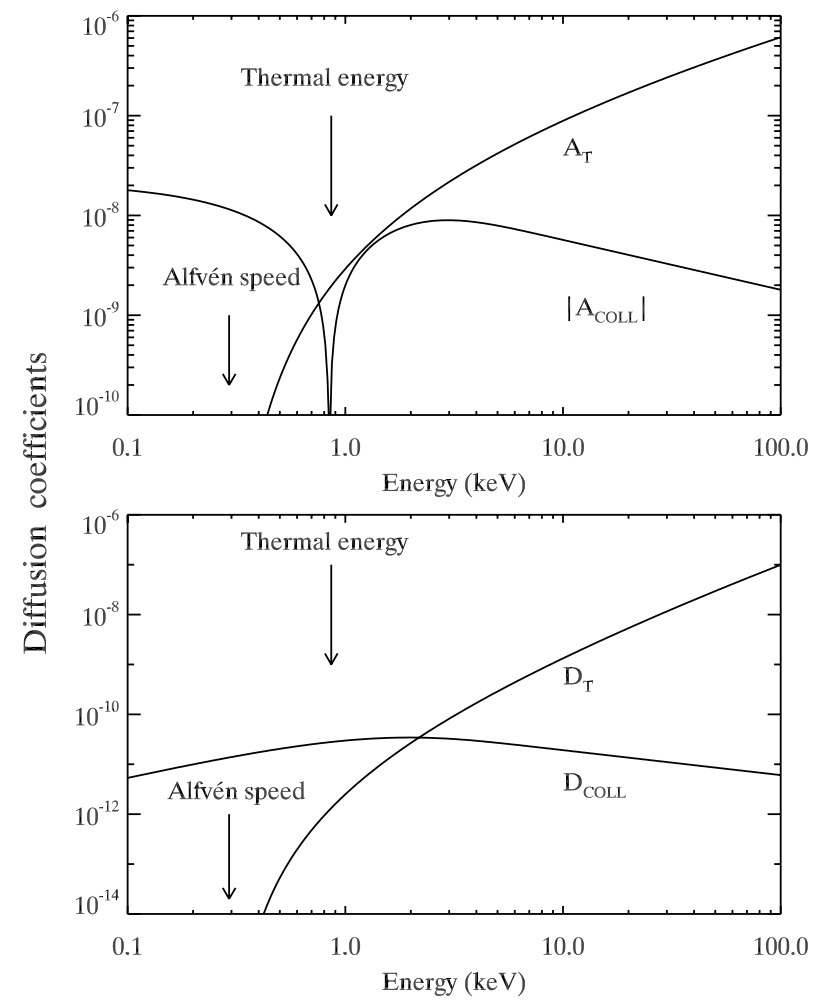

Fig. 3. Upper panel: the absolute value of the dimensionless Coulomb (subscript ${ }_{\text {COLL }}$ ) and transit-time damping (subscript ${ }_{\mathrm{T}}$ ) convection coefficients. Lower panel: the dimensionless Coulomb and transit-time damping diffusion coefficients. The arrows mark the thermal energy of the electrons in the plasma and the kinetic energy of electrons moving with the Alfvén speed $v_{\mathrm{A}}$.

of change of the density is $\mathrm{d} n / \mathrm{d} t=n \cdot v / L$. Defining the escape time $\tau=L / c$ yields Eq. (23), where the factor $T_{\mathrm{H}}=\Omega_{\mathrm{H}}^{-1}$ comes from the transformation of the equation into dimensionless form.

In a more general case, where collisions have an effect, the particles cannot stream freely out of the accelerator, and they will need a longer time to escape. In this case, the escape time $\tau$ can be larger than $L / c$ and the energy dependence on the collision frequencies will cause $\tau$ to vary with the energy. Since we do not address here the physical details of the escape we will assume for now that $\tau(E)$ is constant for all energies.

Under the influence of collisions, we are not allowed to use $L$ as the length of the path traversed by a particle before it can leave the accelerator: $L(E)=c \tau(E)$ denotes the effective length for particle escape. It is best to think of the escape time $\tau$ as a general parameter describing the strength of the particle trapping in the accelerator: the trapping becomes the more efficient, the larger $\tau$.

The source term $Q(E)$ is needed to keep the number of particles in the accelerator constant. Physically, these are electrons supplied by a return current, needed to keep electrically neutral the region where the electrons are accelerated. We assume that the electrons supplied by the return current mechanism are "cool" in the sense that their distribution is comparable with a thermal spectrum and with the temperature of the ambient plasma. Therefore we have

$Q=\dot{n}_{0} \cdot N_{\mathrm{MB}}(E)$,

where

$N_{\mathrm{MB}}(E)=2 \pi^{-1 / 2}\left(k_{\mathrm{B}} T\right)^{-3 / 2} \sqrt{E} \mathrm{e}^{-E /\left(k_{\mathrm{B}} T\right)}$ 
is the Maxwell-Boltzmann distribution normalized to unity, and $\dot{n}_{0}=\int S N \mathrm{~d} E$ is the rate of escaping particles.

\subsection{Method of solution}

Equation (13) is solved numerically using the Crank-Nicolson finite differences scheme in logarithmic energy space, which is well suited for diffusion problems. This method is the same as used in MLM and gives accurate results with fixed steps in time and $\log E$.

\subsection{From electrons to photons}

The numerical solution described above yields electron spectra. For a meaningful comparison with the observations we need photon spectra. They are generated by bremsstrahlung from the electron distribution. Since the model spectra are in equilibrium (particle losses by escape and coulomb collisions are compensated by the acceleration) a thin-target emission is computed.

We convert the energy differential electron flux distribution $F(E)$ in electrons $\mathrm{cm}^{-2} \mathrm{~s}^{-1} \mathrm{keV}^{-1}$ into the photon spectrum $J(\varepsilon)$ observed at Earth in photons $\mathrm{cm}^{-2} \mathrm{~s}^{-1} \mathrm{keV}^{-1}$ using the equation for the thin-target bremsstrahlung emission:

$J(\varepsilon) \mathrm{d} \varepsilon=\frac{n V}{4 \pi R^{2}} \int_{\varepsilon}^{\infty} N(E) \mathrm{d} \sigma(\varepsilon, E) \mathrm{d} E$,

where $V$ is the source volume and $R$ is the distance from the Sun. The cross section $\mathrm{d} \sigma(\varepsilon, E)$ used is the fully relativistic, spatially integrated Bethe-Heitler formula (Bethe \& Heitler 1934) without further approximations. This should adequately represent the emission process of the computed electron spectrum in a looptop source.

We will consistently use the notation $\delta, \gamma, F_{E_{0}}, J_{\varepsilon_{0}}, E_{*}, \varepsilon_{*}, F_{*}$ and $J_{*}$ for, respectively, the electron spectral index, the photon spectral index, the electron density at energy $E_{0}$, the photon flux at energy $\varepsilon_{0}$, the electron pivot-point energy, the photon pivotpoint energy, the electron density at $E_{*}$ and the photon flux at $\varepsilon_{*}$.

\section{Results}

Section 4.1 presents the detailed properties of the numerical solution of Eq. (13) for the standard set of parameters ("default values" given in Table 2) and the energy independent escape model. In a second step (Sect. 4.2) we will proceed to study the dependence of the results on the values of the model parameter and, finally, examine an alternative escape model (Sect. 4.3).

\subsection{Results for the default values of the model parameters}

In this section the default values for the model parameters presented in Table 2 are used. In this case, $\tau$ and $I_{\mathrm{ACC}}$ are the only free parameters of the model, and the acceleration coefficients are much larger than the Coulomb collisional coefficients in the energy range above $10 \mathrm{keV}$ (see Fig. 3). Therefore, we expect that the electron spectrum in this energy range depends only on the value of the product $I_{\tau}=I_{\mathrm{ACC}} \cdot \tau$ (cf. Eq. (13)), and we can basically work with one free parameter. For some choices of the model parameters, this approximation will not be valid anymore, so we will relax this assumption in Sect. 4.2.

Figure 4 presents the electron spectra resulting from the numerical solution of Eq. (13) for 4 different values of $I_{\tau}$, which yield different values of the electron spectral index $\delta$ fitted in the

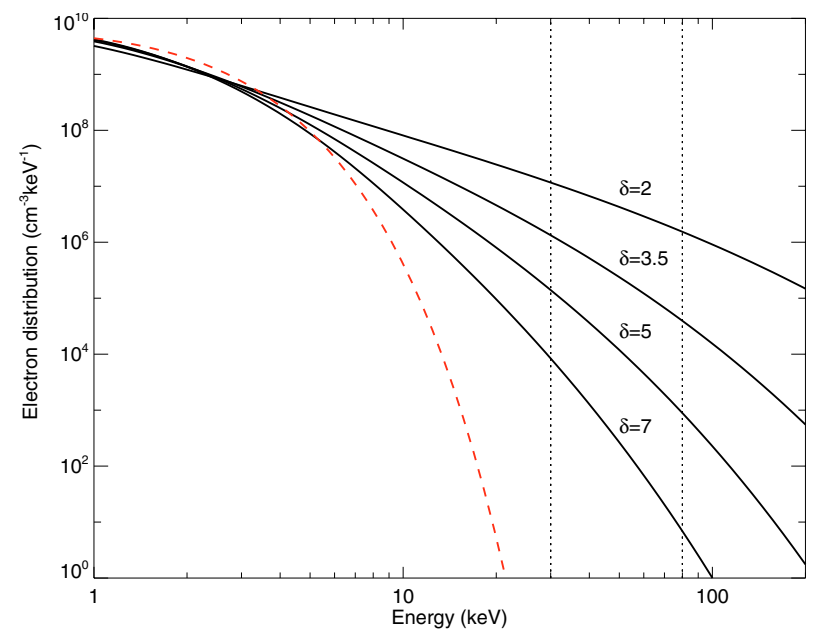

Fig. 4. Accelerated electron distributions with different values of the power-law index resulting from changes in $I_{\tau}=I_{\mathrm{ACC}} \cdot \tau$. The dashed curve represents the ambient Maxwellian distribution. The two dotted lines indicate the energy range used for the computation of the powerlaw index $\delta$ shown above each spectrum.

energy range $30-80 \mathrm{keV}$. The harder spectra are the ones resulting from a higher value of $I_{\tau}$. The dependence of the spectral index on $I_{\tau}$ is shown in the top panel of Fig. 5. Note that in the case where acceleration is weak or escape strong (that is, $I_{\tau}$ is small) very soft spectra are produced. For increasing trapping and acceleration efficiency $\left(I_{\tau}\right.$ larger) the spectra become harder. The middle panel of Fig. 5 shows the dependency of the electron flux at $50 \mathrm{keV}, F_{50}$, on $I_{\tau}$. Here the flux is larger for larger $I_{\tau}$. During a flare, $I_{\tau}$ will change as more energy is injected into turbulence waves, and therefore both $\delta$ and $F_{50}$ will change. This explains qualitatively the soft-hard-soft effect: as $I_{\tau}$ increases, $\delta$ will decrease and $F_{50}$ will increase. It is shown in the bottom panel of Fig. 5, where $\delta$ vs. $F_{50}$ are plotted as a function of the parameter $I_{\tau}$.

Since $I_{\tau}$ contains the physics of acceleration and escape, the time evolution of the spectral index and flux in our model is a direct consequence of the changes in $I_{\tau}$. For example, if the energy density of the turbulent waves grows, reach a maximum value and then decreases, the electron spectrum will harden until peak time and soften again.

To check the quality of the numerical solution, we obtain an approximated analytical expression for the function $\delta\left(I_{\tau}\right)$, whose computation is explained in Appendix A. The approximate curve is plotted in the top panel of Fig. 5 as a dashed line and agrees well with the numerical solution. Because of the linear nature of Eq. (13), the method exploited in Appendix A cannot be used to get an approximate solution for $F_{50}\left(I_{\tau}\right)$.

The dashed line in the bottom panel of Fig. 5 represents the best fit of a logarithmic function (linear in log-lin space) to the data for $\delta$ in the range 2-8. Such a relation between the electron flux at a reference energy and the spectral index indicates the presence of a pivot point in the electron spectra, as shown in Sect. 2. From the slope and normalization of the dashed line we can get values of the pivot-point energy $E_{*}$ and flux $F_{*}$ for the electron distributions.

More precisely, if

$\delta=a \log F_{E_{0}}+b$,

then

$E_{*}=E_{0} \mathrm{e}^{1 / a} \quad$ and $\quad F_{*}=\mathrm{e}^{-b / a}$ 

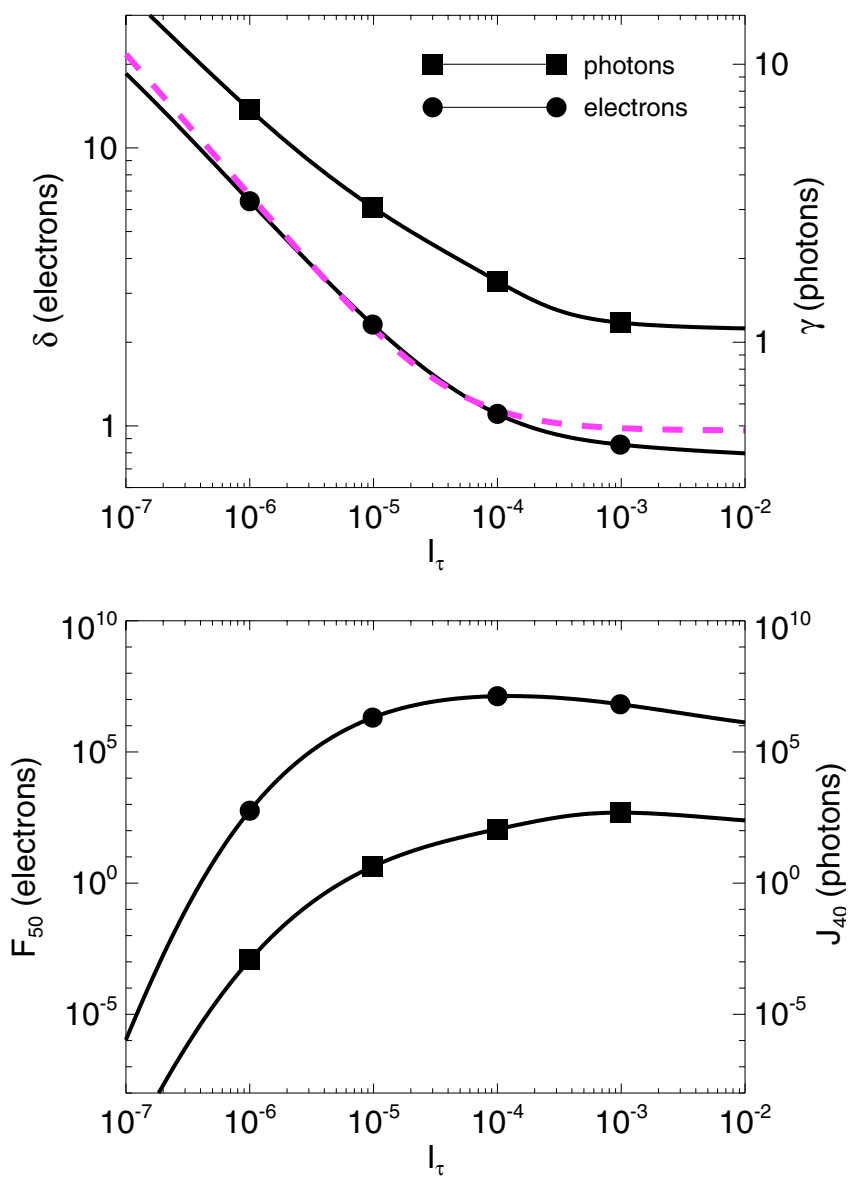

$\mathrm{J}_{40}$ (photons)

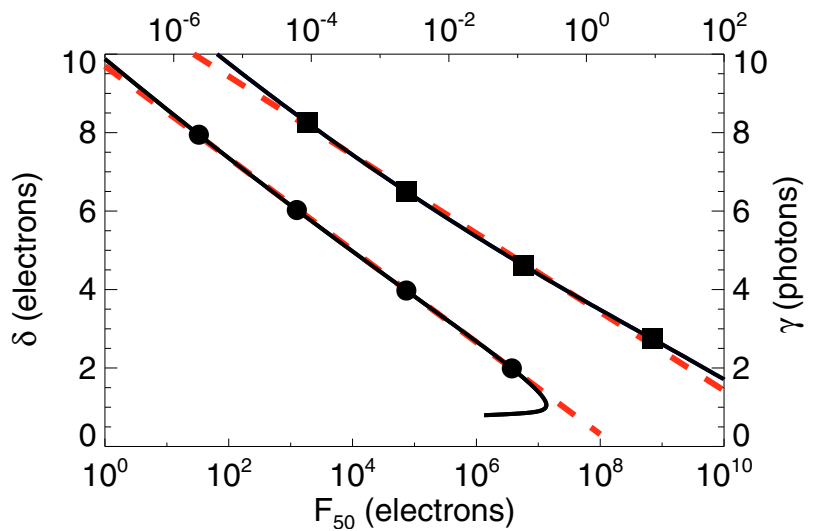

Fig. 5. Top: the electron spectral index $\delta$ (measured in the energy range $30-80 \mathrm{keV}$ ) and the photon spectral index $\gamma$ (measured in the energy range 30-50 keV) plotted against $I_{\tau}$. The dashed line represent the approximated analytical solution computed in Appendix A. Middle: the electron density at $50 \mathrm{keV} F_{50}$ and photon flux at $40 \mathrm{keV} J_{40}$ plotted against $I_{\tau}$. Bottom: $\delta$ vs. $F_{50}$ and $\gamma$ vs. $J_{40}$ plotted parametrically as a function of $I_{\tau}$. The dashed line are the best log-lin fit for $\delta$ in the range 2-8 and for $\gamma$ in the range 3-9.

as follows from Eq. (4). The dashed line in Fig. 5, bottom, corresponds to $E_{*}=7.65 \mathrm{keV}$ and $F_{*}=2 \times 10^{8}$ electrons $\mathrm{cm}^{-3} \mathrm{keV}^{-1}$.

For a meaningful comparison of these results with the observations, a value for the pivot point of the photon spectra is needed. $J_{*}$ and $\varepsilon_{*}$ can be computed by fitting the regression line of $\gamma$ vs. $\log J_{\varepsilon_{0}}$, in the same way as $E_{*}$ and $F_{*}$. Note that while $J_{*}$ depends on the source volume, $\varepsilon_{*}$ can be immediately compared with the observations. Using the photon spectral index computed in the energy range $30-50 \mathrm{keV}$, the regression (restricting $\gamma$ to the range 3-9) yields the pivot-point coordinates $\varepsilon_{*}=5.0 \mathrm{keV}$ and $J_{*}=1.9 \times 10^{3} \cdot V_{27}$ photons $\mathrm{cm}^{-2} \mathrm{~s}^{-1} \mathrm{keV}^{-1}$, where $V_{27}$ is the volume of the source in units of $10^{27} \mathrm{~cm}^{3}$.

The above value of the pivot-point energy is too low compared to the looptop source observations by Battaglia \& Benz (2006) summarized in Table 1 , reporting $\varepsilon_{*} \approx 20 \mathrm{keV}$. This means that if the photon spectral index of our model varies from, say, 3 to 6 , the non-thermal flux is subjected to an excursion which is too large to account for the observed behavior of the spectrum in solar flares.

Two possible solutions of this problem exist: we can explore the parameter space to find out if there is some combination of the parameter values which indeed produces a higher pivot-point energy $\epsilon_{*}$, or we can try to modify the escape term.

\subsection{Exploration of the parameter space}

The set of default parameters used in the previous section yields a pivot-point energy $\varepsilon_{*}$ which is lower than the observed value. However, the pivot-point energy depends on model parameters like $T, n$ etc. Therefore a better coverage of parameter space than the example reported above is needed to assess the range of variability of $\varepsilon_{*}$. The pivot-point energy depends on the temperature of the ambient plasma $T$, its electron density $n$ and its magnetic field strength $B_{0}$ (indirectly, through the Alfvén speed). We therefore compute the equilibrium solutions of Eq. (13) for a large number of combinations of different values of these parameters, each set yielding a value for $\varepsilon_{*}$. In this way we sample the function $\varepsilon_{*}(T, n, \cdots)$.

There is however a complication: for some choices of the parameters, the approximation that the spectral index and flux depend only on $I_{\tau}=\tau \cdot I_{\mathrm{ACC}}$ will not be valid anymore. This approximation becomes invalid if $A_{\mathrm{COLL}} / A_{\mathrm{T}}$ or $D_{\mathrm{COLL}} / D_{\mathrm{T}}$ approach unity. We note that both ratios are roughly proportional to $n^{2}$ at high energies, and therefore the approximation becomes bad for large values of the density. In this case, the escape time $\tau$ and the acceleration parameter $I_{\mathrm{ACC}}$ separately influence the spectral index and flux, and the pivot-point energy will depend on the $\tau$ vs. $I_{\mathrm{ACC}}$ relation. Since we have not modeled the physical mechanism responsible for the trapping of particles, the details of how $\tau$ depends on $I_{\mathrm{ACC}}$ are not known.

We estimate the range of pivot points by choosing several representative paths in the $\tau, I_{\mathrm{ACC}}$-plane (as shown in Fig. 6) and computing the pivot-point coordinates resulting from spectra computed along these paths.

Recalling that in first approximation the spectral index depends only on the product $I_{\tau}=I_{\mathrm{ACC}} \cdot \tau$, the paths are required to start from a point on the line $\tau \cdot I_{\mathrm{ACC}}=10^{-7}$ and end in a point on the line $\tau \cdot I_{\mathrm{ACC}}=10^{-4}$. The region between these two delimitation lines is shown in gray in Fig. 6. These limits are chosen to ensure that the computed photon spectral indices fall into the observed range. For the standard values of the parameters, the region above the gray area yields spectral indices harder than about 1.5 and the region below yields $\gamma$ softer than about 15 .

There are however more constraints on the values of $I_{\mathrm{ACC}}$. This parameter is the product of the dimensionless wave number and the energy density in the turbulent fast mode waves. In our simplified version of transit-time damping acceleration, the time evolution of the spectral energy distribution of the waves is not computed. Therefore we set the maximum value of $I_{\mathrm{ACC}}$ at $I_{\mathrm{ACC}}^{\mathrm{MAX}}=6 \times 10^{-4}$. It is the maximum value that is obtained by MLM during the cascading process (read from the plot in Fig. $6 \mathrm{~b}$ 


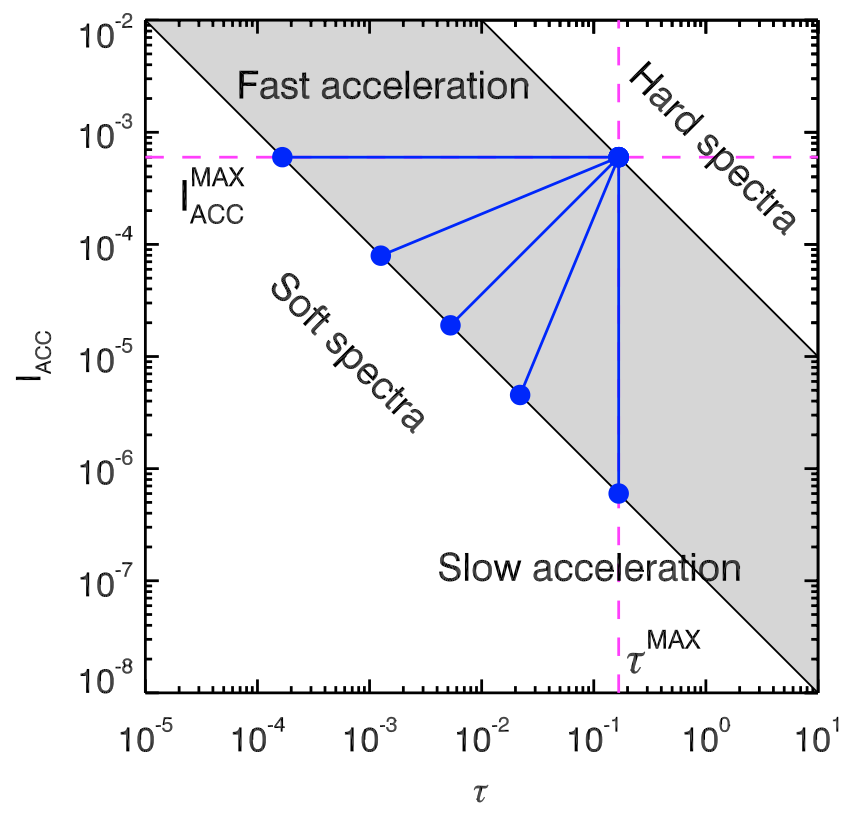

Fig. 6. Five paths (between filled circles) in the $\tau, I_{\mathrm{ACC}}-$ plane as described by Eq. (29), corresponding to $\arctan \alpha=0, \pi / 4, \pi / 2,3 \pi / 4, \pi / 2$. The gray shaded region contains the points yielding photon spectra with a spectral index lying approximatively in the 1.5-15 range. The two dashed lines have abscissa and ordinate of $\tau^{\mathrm{MAX}}$ and $I_{\mathrm{ACC}}^{\mathrm{MAX}}$, respectively.

in MLM). Since $I_{\mathrm{ACC}}$ controls the acceleration efficiency, values which are much smaller then $I_{\mathrm{ACC}}^{\mathrm{MAX}}$ yield a weak and slow acceleration, contrary to the observations. Larger values of $I_{\mathrm{ACC}}$ could result from a higher energy density in the waves, but in that regime the wave amplitude becomes so large that nonlinear effects are likely to a lead to a breakdown of the physical model used by MLM to compute the acceleration coefficients. Thus values of $I_{\mathrm{ACC}}$ much larger than $I_{\mathrm{ACC}}^{\mathrm{MAX}}$ do not necessarily improve the acceleration efficiency.

For these reasons we additionally require that all the paths end on the point $\left(\tau^{\mathrm{MAX}}, I_{\mathrm{ACC}}^{\mathrm{MAX}}\right)$, where $\tau^{\mathrm{MAX}}$ is the point on the upper delimitation line with ordinate $I_{\mathrm{ACC}}^{\mathrm{MAX}}$. Furthermore, we also require the paths to be monotonically increasing functions of both coordinates (this guarantees that the product $I_{\tau}$ is also monotonically increasing). Our reference paths are thus given by

$I_{\mathrm{ACC}}=I_{\mathrm{ACC}}^{\mathrm{MAX}}\left(\frac{\tau}{\tau^{\mathrm{MAX}}}\right)^{\alpha}$

for different values of $\alpha$ between 0 and $\infty$. They represent straight lines in the logarithmic $I_{\mathrm{ACC}}$ vs. $\tau$ plot shown in Fig. 6 , intersecting in the point with coordinates $\left(\tau^{\mathrm{MAX}}, I_{\mathrm{ACC}}^{\mathrm{MAX}}\right)$.

The results are shown in Fig. 7, where the value of the pivotpoint energy $\epsilon_{*}$ is plotted for 3 different values of the plasma temperature $(T=10,20,30 \mathrm{MK})$ and several different values of the plasma density between $n=10^{9} \mathrm{~cm}^{-3}$ and $n=10^{11} \mathrm{~cm}^{-3}$. The different points for each temperature and density represent the range of variation of $\epsilon_{*}$ for different values of $\alpha$ as explained above. We have used $\alpha=0, \sqrt{2}-1,1, \sqrt{2}+1, \infty$.

The pivot-point energy increases with temperature. The density dependence of the pivot-point energy is weak, while the pivot-point flux decreases at higher densities. The number of accelerated particles becomes very small at densities of $10^{12} \mathrm{~cm}^{-3}$ and higher, because the particles lose energy in the collisions faster than they gain energy from the waves. The spread of the pivot-point energies shown in Fig. 7 increases at higher densities,

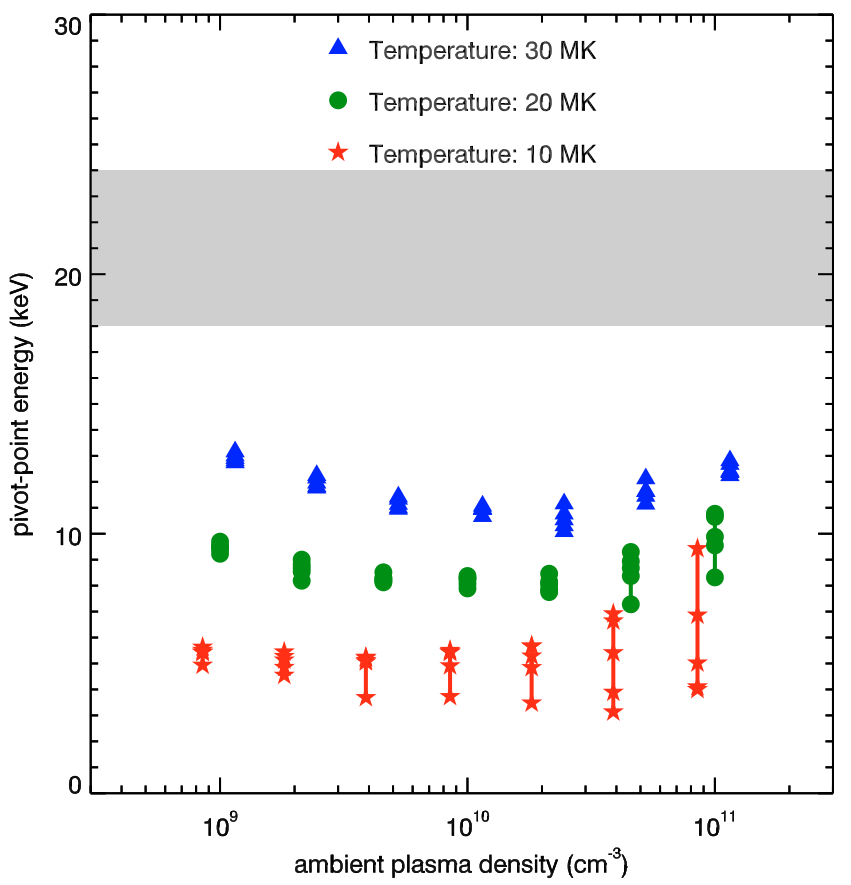

Fig. 7. Dependance of the photon pivot-point energy $\epsilon_{*}$ on the electron density $n$ for plasma temperatures $T$ of $10 \mathrm{MK}$ (stars), $20 \mathrm{MK}$ (circles) and $30 \mathrm{MK}$ (triangles). The density values of the 10 and $30 \mathrm{MK}$ data points have been shifted by $15 \%$ to avoid overlap with the other points. The spread arise from computation of the spectra along different paths as explained in the text. The gray shaded region represents the observed range of values of $\epsilon_{*}$ reported in Table 1.

as expected from the high-density breakdown of the approximation that the pivot point only depends on $I_{\mathrm{ACC}} \cdot \tau$.

\subsection{Alternative escape modeling}

As an alternative way for explaining the high values of $\varepsilon_{*}$, we now modify the escape mechanism. For instance, if an electric potential $V_{\mathrm{E}}$ is present between the accelerator and the footpoints, electrons with kinetic energy lower than the threshold energy $E_{\mathrm{T}}=e V_{\mathrm{E}}$ will not be able to cross that barrier and will therefore not leave the accelerator. Such an electric field is expected to exist and drive the return current of electrons from the chromosphere. In this scenario the trapping is ideal below $E_{\mathrm{T}}$, and $\tau$ becomes infinitely large.

This means that the escape time is given by:

$\tau(E)=\left\{\begin{array}{ccc}\infty & \text { if } & E \leq E_{\mathrm{T}} \\ \tau & \text { if } & E>E_{\mathrm{T}}\end{array}\right.$

The results are shown in Fig. 8 for two values of $E_{\mathrm{T}}, 25 \mathrm{keV}$ and $45 \mathrm{keV}$. Note that the value of the pivot-point energy $\varepsilon_{*}$ increases with increasing $E_{\mathrm{T}}$. The pivot point energies reach the observed values for $E_{\mathrm{T}} \simeq 30 \mathrm{keV}$, although even at $45 \mathrm{keV}$ some paths in the $\tau, I_{\mathrm{ACC}}-$ plane deliver lower pivot-point energies than observed.

The points in Figs. 7 and 8 with larger pivot-point energy correspond to the paths with the lowest values of $\alpha$, where the escape time $\tau$ changes faster than the acceleration parameter $I_{\mathrm{ACC}}$.

A comparison of observed spectra of a looptop source (for the M 3 flare of December 4, 2002) and the spectra computed by the stochastic acceleration model with $E_{\mathrm{T}}=40 \mathrm{keV}$ is shown in Fig. 9. The model parameters used were temperature $T=$ $20 \mathrm{MK}$ and density $n=5 \times 10^{10} \mathrm{~cm}^{-3}$ and $\alpha=\sqrt{2}-1$, yielding 

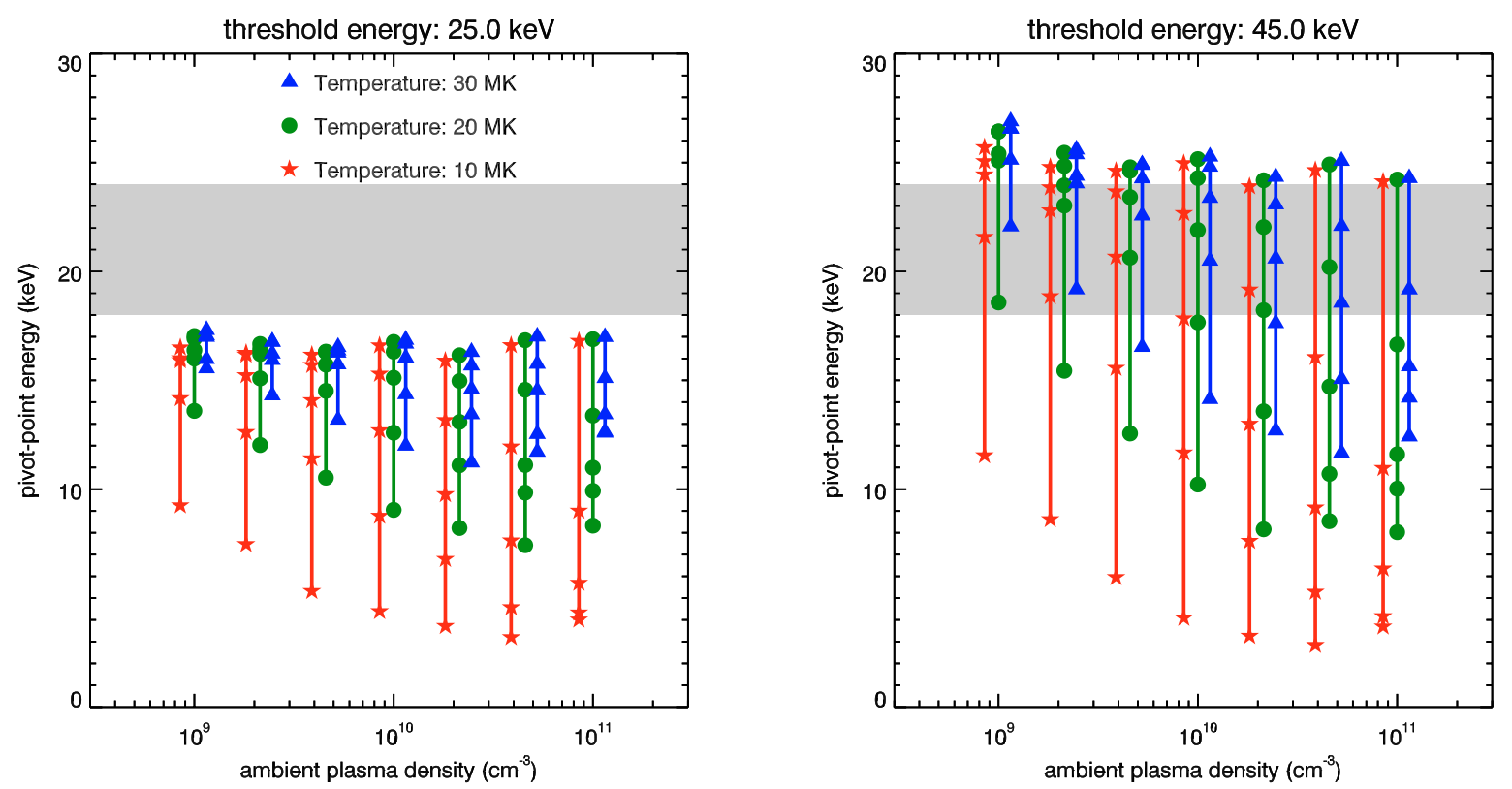

Fig. 8. Same as in Fig. 7, but with a modified escape term featuring perfect trapping below $25 \mathrm{keV}$ (left panel) and below $40 \mathrm{keV}$ (right panel).
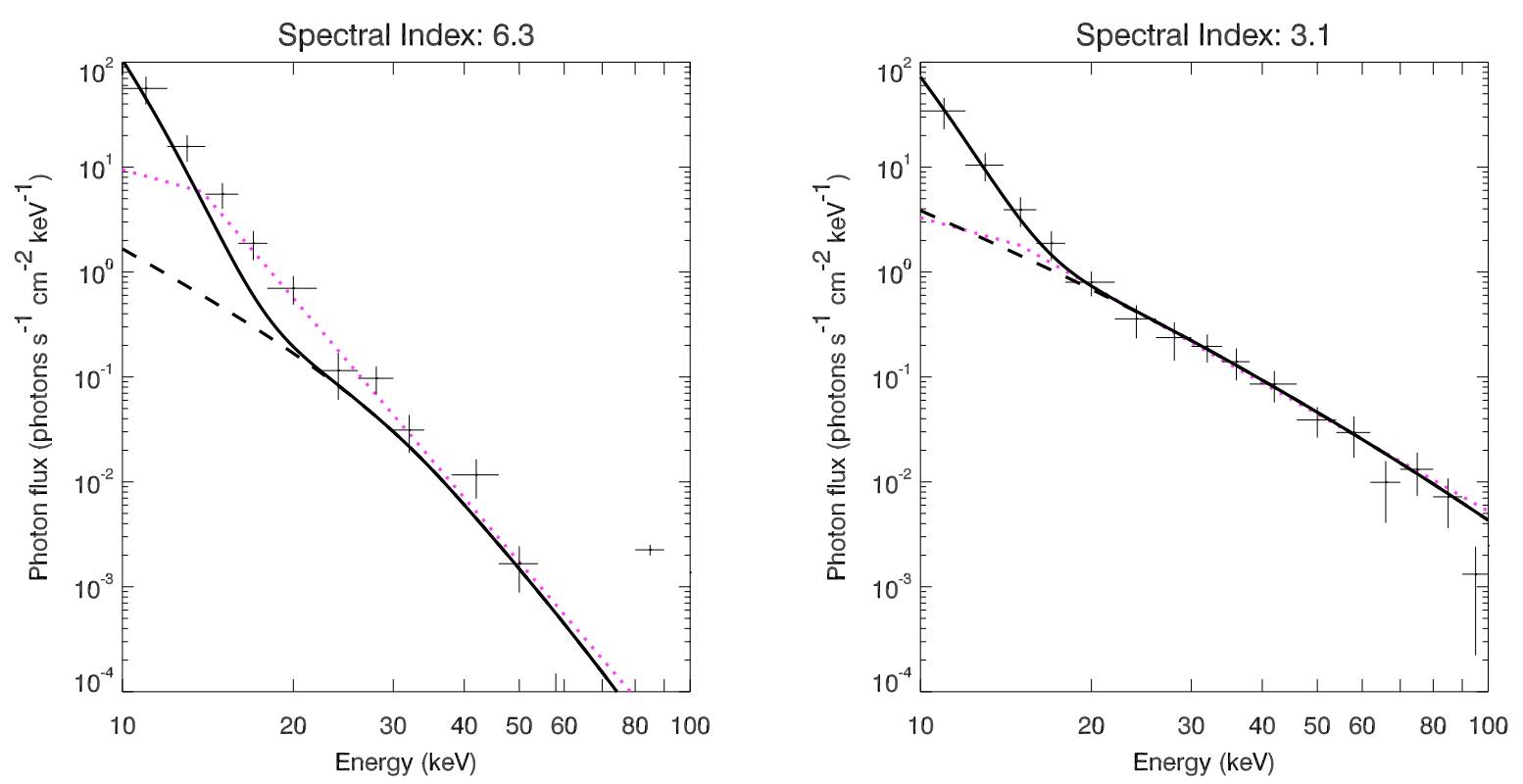

Fig. 9. Looptop source spectra observed during the M 3 flare of December 4, 2002, represented by crosses (bin width and error bar, data courtesy of M. Battaglia). The dotted line is a power-law fit to the data (a low-energy turnover at around $15 \mathrm{keV}$ ) with spectral index $\gamma$ of 6.3 for the left panel and 3.1 for the right panel. In each panel, the dashed line is a stochastic acceleration model spectrum computed for model parameters: temperature $T=20 \mathrm{MK}$, density $n=5 \times 10^{10} \mathrm{~cm}^{-3}$, and threshold energy $E_{\mathrm{T}}=40 \mathrm{keV}$ (chosen such that the pivot-point energy $\varepsilon_{*}$ matches the observed value of $\sim 18 \mathrm{keV}$ reported in Battaglia \& Benz 2006). The continuous line is the sum of the model spectrum with an additional isothermal component, needed to fit the low-energy range of the spectra.

a pivot-point energy $\epsilon_{*}=18.7 \mathrm{keV}$, similar to observed value of $18.1 \mathrm{keV}$ reported by Battaglia \& Benz (2006). The model spectra are represented by the dashed lines, the power-law fit to the data by the dotted line, and the sum of the model spectra with an isothermal component by the continuous line.

In our model, the value of the total photon flux observed at earth depends linearly on the accelerator volume, which acts as a normalization factor for the model spectra. For the comparison with the observed data, the volume can be freely chosen, but is assumed not to change along the path in the $\tau, I_{\mathrm{ACC}}$-plane. In practice, that means that the volume can be chosen in order to match the data (or the power-law fittings) at, say, peak time, and automatically the spectra at all the times during the emission peak will match, provided that the pivot-point energies of the observed and the model spectra are the same.

The low energy part of the observed spectrum shows a thermal emission much larger than the model spectra emission. This can be understood if particle acceleration takes place in a smaller volume than the one where the thermal emission takes place. For the events shown in Fig. 9, the filling factor of the accelerator amounts to around $10^{-3}$.

The left panel of Fig. 9 shows a mismatch between the observed data and the model spectra below $30 \mathrm{keV}$. This is due to the effect of the escape model used: the suppression of 
electron escape results in a hardening of the electron spectra below $E_{\mathrm{T}}=40 \mathrm{keV}$. Particles with energy lower than $E_{\mathrm{T}}$ are able to extract more energy out of the waves until they are accelerated beyond $E_{\mathrm{T}}$. A turnover occurs at around $0.7 E_{\mathrm{T}}$ for photon spectra, due to the shape of the bremsstrahlung cross section $\mathrm{d} \sigma(\varepsilon, E)$.

\section{Discussion}

The important results are the following:

- The transit-time damping mechanism with escape is able to provide photon spectra with spectral indices in the deka-keV energy range corresponding to the observed range for flares ( $\gamma=2$ to 10, Dennis 1985; Battaglia et al. 2005) as the ratio between the trapping and accelerating efficiency is varied (Fig. 4). This confirms that escape indeed does soften the spectrum for different values of densities and temperatures of the accelerator.

- Assuming constant values of temperature and density in the accelerator (as may be the case during a hard X-ray peak with a duration of some tens of seconds) we are able to produce a correlation between the photon spectral index and flux yielding an approximate pivot point in photon flux vs. energy space (Figs. 5 and 7).

- The energy of the pivot point in the simplest model is around $10 \mathrm{keV}$ and is lower than the reported observed value of $20 \mathrm{keV}$.

- An additional increase of the strength of trapping below 20-30 keV (as may be obtained from the presence of a potential barrier) increases the pivot-point energy to $20 \mathrm{keV}$ (Fig. 8).

- The potential barrier hardens the spectrum below its threshold energy. For large $\gamma$, this flattens the spectrum more than observed in the range where the non-thermal emission still dominates the thermal emission (Fig. 9, left).

- The observational upper limit for the energy of a turnover in the photon spectra lies around $15 \mathrm{keV}$ (Saint-Hilaire \& Benz 2005). Model spectra computed for threshold energies below about $23 \mathrm{keV}$ yield a turnover lower than $15 \mathrm{keV}$ and pivot-point energies below $16 \mathrm{keV}$.

What is the physical significance of the pivot point? We would like to point out first that there is not necessarily any: it can be thought of as a useful parameter which describes the variation in flux and spectral index of the observed spectra, and one has to explain this variation first in the context of a model such as done in this work. However, the fact that it appears at an energy which is comparable to the estimated turnover energy of the non-thermal component, as well as the energy below which the thermal component dominates is a suggestive fact.

The model suggests that the main factor influencing the pivot-point energy is the number of electrons available at energy comparable with it. We propose that, in general, the presence of a large supply of electrons at some energy implies a similar energy for the pivot point, on the condition that the electron collisional energy losses are negligible above that energy range, such that the acceleration process can effectively energize that population. It is not important if such a population exist at the beginning of the acceleration, but it must be present in the later stages and be maintained until the equilibrium is reached.

\section{Conclusions}

The model studied assumes that turbulent fast-mode waves are present in the accelerator region. The equilibrium spectrum of electrons subjected to Coulomb collisions and transit-time damping interactions with the waves are computed allowing for trapping/escape and replenishment of "cold" particles. As such the model does not distinguish between the accelerator and the radiator and considers the photon spectra emitted by the electrons in the accelerator itself. The relatively dense looptop sources observed by RHESSI fit this scenario as accelerators/emitters, and the observed high densities support a scenario in which trapping plays an important role in acceleration. Therefore, spectral observations of these looptop sources may well deliver a snapshot of the electrons in the accelerator.

To match the low-energy part of the observed spectrum, we have to add an additional thermal component at the observed temperature. This component fills a much larger volume (factor of 1000). The heating may have occurred by earlier acceleration or may be the result of waves escaping from the accelerator region with energy density too low to yield significant energy gain per particle.

The goals of this work were to test whether a stochastic acceleration mechanism can account for the observed soft-hardsoft behavior and, in particular, to find a minimal set of modifications to the transit-time damping mechanism yielding a pivotpoint like behavior of the electron spectrum in the deka-keV range. Therefore we have not fully investigated the physics of escape, and in particular we leave open the question of the connection between the acceleration process and the trapping mechanism.

The results shown in Sect. 4 confirm that the hardness of the spectrum of accelerated particles in a stochastic acceleration model depends strongly on particle trapping. This can be understood by recalling that stochastic acceleration can be thought as a transport of particles in energy space due to a random walk process. While acceleration pushes particles toward higher energies, the escape acts against this flow, because fast particles are more likely to be lost, and replaced by particles at lower energies. Therefore, when the losses are stronger, the average time a particle spends random-walking in energy space is shorter, thus gaining less energy. This results in softer spectra.

We have used one specific kind of stochastic acceleration model and a simple escape scenario, but the physical mechanisms explored by the specific models are general enough that other stochastic models and escape terms are expected to follow the same general behavior leading to a soft-hard-soft effect, manifesting itself as a correlation of the time evolution of the spectral index and the non-thermal X-ray flux.

A simple escape model cannot account for the observed value of the photon pivot-point energy, which is about a factor 2 higher. The modification in the escape term featuring no escape below a threshold energy $E_{\mathrm{T}} \simeq 40 \mathrm{keV}$ can increase the energy of the pivot point to the observed values, but at the price of introducing a spectral hardening below $30 \mathrm{keV}$, which is not observed for large $\gamma$.

One possible interpretation of the threshold energy used in the alternative escape model is the presence of an electric field driving the return current. It was treated as a free parameter, but physically the escape process and the return current are linked by the electric conductivity of the loop legs. In this sense the model is not self consistent. The issues of transport of the escaped particles to the footpoint and return currents are important (see for 
example Zharkova \& Gordovsky 2005) and need to be addressed in future work to be able to derive footpoint spectra.

Thus the model is not able to account quantitatively for all the observed features of the intricate spectral behavior of the hard X-ray emission from looptop sources of solar flares. Nevertheless, the model qualitatively accounts for the key features of the spectral evolution, going one step further toward the solution of the acceleration problem. This allows to interpret the impressive RHESSI observations showing spectral variation in looptop sources.

The simplifications introduced by the model may well be responsible for its shortcoming in reproducing the observed spectra for large $\gamma$. Future improvements may include an isotropization process and magnetic trapping. This will require the extension of the model including at least one spatial dimension and the pitch angle distribution of the particles.

Acknowledgements. We thank M. Battaglia for sharing her results before publication, and K. Arzner, S. Bruderer, L. Vlahos and the participants of the theoretical working group at the 6th RHESSI Workshop for useful discussions. The analysis of RHESSI data at ETH Zurich is partially supported by the Swiss National Science Foundation (grant no. 20-67995.02). This research has made use of NASA's Astrophysics Data System Bibliographic Services.

\section{Appendix A: Approximate analytical solution}

We compute here an approximate analytical solution in the energy range $k T /\left(m c^{2}\right) \ll E \ll 1$. In this range, we can neglect the influence of the Coulomb collisional coefficients $A_{\mathrm{C}}$ and $D_{\mathrm{C}}$ as well as the source term $Q$. In equilibrium Eq. (13) becomes:

$\frac{D_{0}}{2} \frac{\partial^{2} N}{\partial E^{2}}+\left(D_{1}-A_{0}\right) \frac{\partial N}{\partial E}+\left(D_{2}-A_{1}-S\right) N=0$,

where the coefficients $A_{i}$ and $D_{i}$ are the factors of order $i$ occurring in the Taylor expansion of $A$ and $D$ around $E$. Their computation is tedious, but straightforward. Neglecting the term of order $\xi^{2}$ and setting $\gamma=1$ we get

$D_{0}=K \beta^{3}(-5 / 4-\log \xi)$

$D_{1}=K \beta(-11 / 4-3 \log \xi)$

$D_{2}=\frac{2 K}{\beta}(-17 / 8-3 / 2 \cdot \log \xi)$

$A_{0}=2 K \beta(-1-\log \xi)$

$A_{1}=\frac{2 K}{\beta}(-1 / 4-\log \xi)$

where $K=\frac{\pi}{8} \beta_{\mathrm{A}}^{2} I_{\mathrm{ACC}}, \xi=\beta_{\mathrm{A}} / \beta$.
The solution $N(E)$ will be approximatively given by a power-law around $E=E_{0}$

$N(E)=N_{0}\left(\frac{E}{E_{0}}\right)^{-\delta}$.

Plugging this function into Eq. (A.1) yields a quadratic equation for $\delta$ :

$c_{2} \delta^{2}+c_{1} \delta+c_{0}=0$

with coefficients

$c_{2}=\frac{\tau D_{0}}{2 E_{0}^{2}}$

$c_{1}=\frac{\tau}{E_{0}}\left(\frac{D_{0}}{2 E_{0}}-D_{1}+A_{0}\right)$

$c_{0}=\tau D_{2}-\tau A_{1}-\sqrt{2 E_{0}}$.

The solution of the quadratic equation yields $\delta$ as a function of $I_{\tau}=I_{\mathrm{ACC}} \cdot \tau$ and the model parameters. The asymptotic behavior of the solution is the following: $\delta$ is constant for $I_{\tau} \rightarrow \infty$, and $\delta$ is proportional to $1 / \sqrt{I_{\tau}}$ for $I_{\tau} \rightarrow 0$.

\section{References}

Aschwanden, M. J. 2002, Space Sci. Rev., 101, 1 Battaglia, M, \& Benz, A. O. 2006, A\&A, 456, 751

Battaglia, M., Grigis, P. C., \& Benz, A. O. 2005, A\&A, 439, 737

Benz, A. O., \& Grigis, P. C. 2002, Sol. Phys., 210, 431

Bethe, H., \& Heitler, W. 1934, Roy. Soc. Lond. Proc. Ser. A, 146, 83

Brosius, J. W., Davila, J. M., Thomas, R. J., \& Monsignori-Fossi, B. C. 1996, ApJS, 106, 143

Dennis, B. R. 1985, Sol. Phys., 100, 465

Emslie, A. G., Kontar, E. P., Krucker, S., \& Lin, R. P. 2003, ApJ, 595, L107

Emslie, A. G., Kucharek, H., Dennis, B. R., et al. 2004, J. Geophys. Res. (Space Phys.), 109, 10104

Grigis, P. C., \& Benz, A. O. 2004, A\&A, 426, 1093

Grigis, P. C., \& Benz, A. O. 2005, A\&A, 434, 1173

Holman, G. D., Sui, L., Schwartz, R. A., \& Emslie, G. A. 2003, ApJ, 595, L97

Kane, S. R., McTiernan, J. M., \& Hurley, K. 2005, A\&A, 433, 1133

Larosa, T. N., \& Moore, R. L. 1993, ApJ, 418, 912

Lin, R. P., Dennis, B. R., Hurford, G. J., et al. 2002, Sol. Phys., 210, 3

Lin, R. P., Krucker, S., Hurford, G. J., et al. 2003, ApJ, 595, L69

Liu, W., Jiang, Y. W., Liu, S., \& Petrosian, V. 2004, ApJ, 611, L53

Masuda, S., Kosugi, T., Hara, H., et al. 1994, Nature, 371, 495

Miller, J. A., Larosa, T. N., \& Moore, R. L. 1996, ApJ, 461, 445

Miller, J. A. 1998, Space Sci. Rev., 86, 79

Petrosian, V., \& Liu, S. 2004, ApJ, 610, 550

Saint-Hilaire, P., \& Benz, A. O. 2005, A\&A, 435, 743

Sui, L., Holman, G. D., \& Dennis, B. R. , 2005, ApJ, 626, 1102

Spitzer, L. 1962, Physics of Fully Ionized Gases (New York: Interscience Publishers)

Trubnikov, B. A. 1965, Rev. Plasma Phys., 1, 105

Zharkova, V. V., \& Gordovsky, M. 2005, The Dynamic Sun: Challenges for Theory and Observations (ESA SP-600), 147.1 